\title{
ARTICLE
}

\section{The discovery of element 117 (INVITED)}

\author{
James B. Roberto* \\ Oak Ridge National Laboratory, Oak Ridge, Tennessee 37831 USA
}

\begin{abstract}
In November 2016, four new chemical elements, nihonium ( $\mathrm{Nh}, \mathrm{Z}=113$ ), moscovium (Mc, $\mathrm{Z}=115)$, tennessine $(\mathrm{Ts}, \mathrm{Z}=117)$, and oganesson $(\mathrm{Og}, \mathrm{Z}=118)$ joined the periodic table. Elements 117 and 118 are the highest atomic numbers $Z$ reached to date, and the $A=294$ isotopes of these elements are the heaviest nuclei ever synthesized. The existence of these elements, together with a consistent trend of increasing lifetimes for superheavy isotopes as neutron number $\mathrm{N}$ increases, provide strong evidence for the long sought "island of stability" for superheavy nuclei as the nuclear shell closure at $\mathrm{N}=184$ is approached. Mc, Ts, and Og were discovered using the hot fusion process, bombarding actinide targets with intense beams of doubly-magic ${ }^{48} \mathrm{Ca}$ ions at the Dubna Gas-Filled Recoil Separator at the Joint Institute for Nuclear Research in Russia. For element 117, a berkelium target from Oak Ridge National Laboratory (ORNL) was required. The berkelium was produced by intense neutron irradiation at ORNL's High Flux Isotope Reactor and chemically separated at the adjoining Radiochemical Engineering Development Center. The ${ }^{249} \mathrm{Bk}+{ }^{48} \mathrm{Ca}$ reaction produced two isotopes of element 117 with atomic weights $A=293$ and 294. These isotopes decayed into ten additional heaviest isotopes of elements $115,113,111,109,107$, and 105, all closer to the predicted island of stability than previously achieved. The discovery of element 117 will be described together with results of other recent experiments in superheavy element research in the context of the critical importance of actinide target materials, implications for the periodic table and the island of stability, and opportunities to synthesize even heavier nuclei, including heavy isotopes of element 118 and new elements 119 and 120.
\end{abstract}

Keywords: actinide; chemical separations; superheavy element; tennessine

\section{Introduction}

Four new chemical elements, nihonium $(\mathrm{Nh})$, moscovium (Mc), tennessine (Ts), and oganesson $(\mathrm{Og})$, with $Z=113,115,117$, and 118 , respectively, officially joined the periodic table in 2016 [1]. Elements 115, 117, and 118 were discovered using the "hot fusion" process [2] by irradiating actinide targets with intense ${ }^{48} \mathrm{Ca}$ ion beams from the U400 cyclotron at the Flerov Laboratory of Nuclear Reactions (FLNR) at the Joint Institute for Nuclear Research (JINR), Dubna, Russia. The resulting nuclear reactions produced compound nuclei (with $\mathrm{Z}=\mathrm{Z}($ actinide $)+\mathrm{Z}(\mathrm{Ca})$ ) that stabilized by emission of excess neutrons. The actinide target materials [3] for these experiments were obtained from unique facilities at Oak Ridge National Laboratory (ORNL) and at the Research Institute of Advanced Reactors (RIAR) at Dimitrovgrad, Russia. The most recent discovery, element 117 [4], required a berkelium target produced at ORNL's High Flux Isotope Reactor (HFIR) and chemically separated and purified at the adjacent Radiochemical Engineering Development Center (REDC). In addition to expanding the periodic table, these discoveries provide evidence for the existence of

*Corresponding author. Email: robertojb@ornl.gov the long sought "island of stability" for superheavy elements proposed by Seaborg in the 1960s.

The term superheavy element generally refers to elements with an atomic number $Z$ of 104 or greater. Cross sections for production of superheavy nuclei using the established "cold fusion" process [5] decline rapidly with increasing $Z$, with the result that discovery of new elements above $Z=113$ is impractical using currently available accelerator facilities. The last element to be discovered using cold fusion was nihonium with $Z=113$, where three nuclei were produced during a multi-year campaign [6] at RIKEN in Japan. The "hot fusion" technique, developed and implemented [5] at FLNR, JINR, resulted in significantly increased production cross sections for higher Z's, enabling expansion of the periodic table to element 118 and perhaps beyond. This technique is dependent upon the availability of neutron-rich actinide targets including ${ }^{244} \mathrm{Pu},{ }^{248} \mathrm{Cm}$, ${ }^{249} \mathrm{Bk}$, and ${ }^{249} \mathrm{Cf}$. These actinides are produced using specialized facilities and expertise available at only a few laboratories worldwide [3]. A summary of new elements discovered since 2000 using actinide $+{ }^{48} \mathrm{Ca}$ reactions is provided in Table 1. Including resulting decay chains, more than 50 new isotopes have been produced in this research. These new elements complete the seventh row of the periodic table. 
Table 1. "Hot fusion" reactions using ${ }^{48} \mathrm{Ca}$ beams on actinide targets have resulted in the discovery of five new elements since 2000 [2].

\begin{tabular}{cccc}
\hline $\begin{array}{c}\text { Element } \\
(\mathrm{Z})\end{array}$ & $\begin{array}{c}\text { Year } \\
\text { first } \\
\text { produced }\end{array}$ & $\begin{array}{c}\text { Actinide } \\
\text { target } \\
\text { material }\end{array}$ & $\begin{array}{c}\text { Nuclei } \\
\text { produced } \\
\text { to date }\end{array}$ \\
\hline $\begin{array}{c}\text { Flerovium } \\
(114)\end{array}$ & 2000 & ${ }^{244} \mathrm{Pu}$ & 99 \\
$\begin{array}{c}\text { Moscovium } \\
(115)\end{array}$ & 2004 & ${ }^{243} \mathrm{Am}$ & 135 \\
$\begin{array}{c}\text { Livermorium } \\
(116)\end{array}$ & 2005 & ${ }^{245,248} \mathrm{Cm}$ & 35 \\
$\begin{array}{c}\text { Tennessine } \\
(117)\end{array}$ & 2010 & ${ }^{249} \mathrm{Bk}$ & 22 \\
$\begin{array}{c}\text { Oganesson } \\
(118)\end{array}$ & 2006 & ${ }^{249} \mathrm{Cf}$ & 5 \\
\hline
\end{tabular}

The existence of superheavy elements is dependent on the details of nuclear shell effects for nuclei with extreme numbers of protons and neutrons. The heaviest stable isotope, ${ }^{208} \mathrm{~Pb}$, has closed shells for both protons and neutrons. The next "doubly-magic" spherical nucleus is expected to occur around $\mathrm{Z}=114-126$ and $\mathrm{N}=184$, the presumed center of the island of stability. It is expected that the influence of these closed shells will confer unusual stability on nuclei in this region, perhaps hundreds or even millions of years. The existence of the island of stability offers the opportunity to study the nuclear and chemical properties of previously unknown elements with extreme numbers of protons, neutrons, and electrons.

\section{Experiment}

\subsection{Production and processing of berkelium}

Actinides in the 10-15 mg quantities required for targets for superheavy element synthesis can only be produced using high-flux reactors. For the synthesis of element 117 using ${ }^{48} \mathrm{Ca}$ beams, a berkelium target is required (combining $Z=20 \mathrm{Ca}$ with $\mathrm{Z}=97 \mathrm{Bk}$ results in a compound nucleus with $Z=117$ ). The ${ }^{249} \mathrm{Bk}$ used for this experiment was produced during a 150-day irradiation of $40 \mathrm{~g}$ of $\mathrm{Am} / \mathrm{Cm}$ cermet microspheres in HFIR at ORNL. The microspheres were loaded into special aluminum tubes and irradiated in the central flux trap of HFIR $\left(2.5 \times 10^{15} \mathrm{n} / \mathrm{s}-\mathrm{cm}^{2}\right)$ during a scheduled campaign to produce ${ }^{252} \mathrm{Cf}$. Cf isotopes (together with smaller amounts of $\mathrm{Bk}$, Es, and $\mathrm{Fm}$ ) are produced through a series of thermal neutron captures and beta decays.

The irradiated $\mathrm{Am} / \mathrm{Cm}$ was transferred to REDC for chemical separations and purification. REDC is a large complex of heavily-shielded hot cells with facilities for remote handling and chemical processing of highly radioactive materials. After allowing several months for the decay of fission products and other short-lived isotopes, the irradiated material was processed using multiple dissolution and ion exchange steps [3,7-11] over several months to extract transcurium elements, including $\mathrm{Cf}$ and $\mathrm{Bk}$. The transcurium products were separated from each other by additional processing resulting in $\sim 200 \mathrm{mg}$ of ${ }^{252} \mathrm{Cf}$ and $\sim 20 \mathrm{mg}$ of ${ }^{249} \mathrm{Bk}$. If desired, 1-2 micrograms of ${ }^{254} \mathrm{Es}$ and picograms of ${ }^{257} \mathrm{Fm}$ can also be recovered. The separated ${ }^{249} \mathrm{Bk}$ (half-life $327 \mathrm{~d}$ ) was further processed and purified to $<10^{-9} \mathrm{~g}{ }^{252} \mathrm{Cf}$ in a glove box and shipped to Dubna. The overall process for production of heavy actinides at HFIR/REDC is summarized in Figure 1.

\subsection{Synthesis of element 117}

Nuclei of element 117 were produced in hot fusion nuclear reactions of ${ }^{48} \mathrm{Ca}$ on ${ }^{249} \mathrm{Bk}$.

$$
{ }^{48} \mathrm{Ca}+{ }^{249} \mathrm{Bk} \rightarrow\left({ }^{297}[117]\right) * \rightarrow{ }^{294,293}[117]+3-4 \mathrm{n}
$$

The result of this reaction is an excited compound nucleus, ${ }^{297} 117$, that stabilizes by the emission of excess neutrons. The most probable reaction channels result in the emission of 3 or 4 neutrons, yielding two isotopes of element 117 with mass numbers 294 and 293, respectively. ${ }^{48} \mathrm{Ca}$ beam energies were $252 \mathrm{MeV}$ and 247 $\mathrm{MeV}$ (corresponding to excitation energies of 39 and 35 $\mathrm{MeV}$, respectively), coinciding with expected maxima in production cross sections for the $3 n$ and $4 n$ channels. The produced isotopes of element 117 subsequently decay by emission of alpha particles to elements 115 , $113,111,109$, etc.

The ${ }^{249} \mathrm{Bk}$ was deposited on Ti foils at the RIAR, and the resulting target foils were mounted on a target wheel at the Dubna Gas Filled Recoil Separator (DGFRS) at FLNR, JINR. The target was irradiated for 140 days, 70 days at each energy, at a ${ }^{48} \mathrm{Ca}$ beam intensity of $\sim 1$ particle microamp.

The target wheel was rotated at $1700 \mathrm{rpm}$ to distribute beam heating. Beam particles were separated from reaction products using the DGFRS, and the reaction products, including nuclei of element 117, were implanted in a solid-state detector. Implantation at the expected energy for an element 117 nucleus followed by an alpha particle emission at the same position with the predicted energy provides evidence of the production of an element 117 nucleus. At this time, the accelerator was turned off, and subsequent alpha decays were monitored under low background conditions. A chain of multiple decays at the expected times and energies from the same position as the original implant and terminated by a fission event provide a unique signature for production of an isotope of element 117. The decay chains for ${ }^{293} 117$ and ${ }^{294} 117$ are illustrated in Figure 2 together with the nuclear landscape for known superheavy isotopes. 


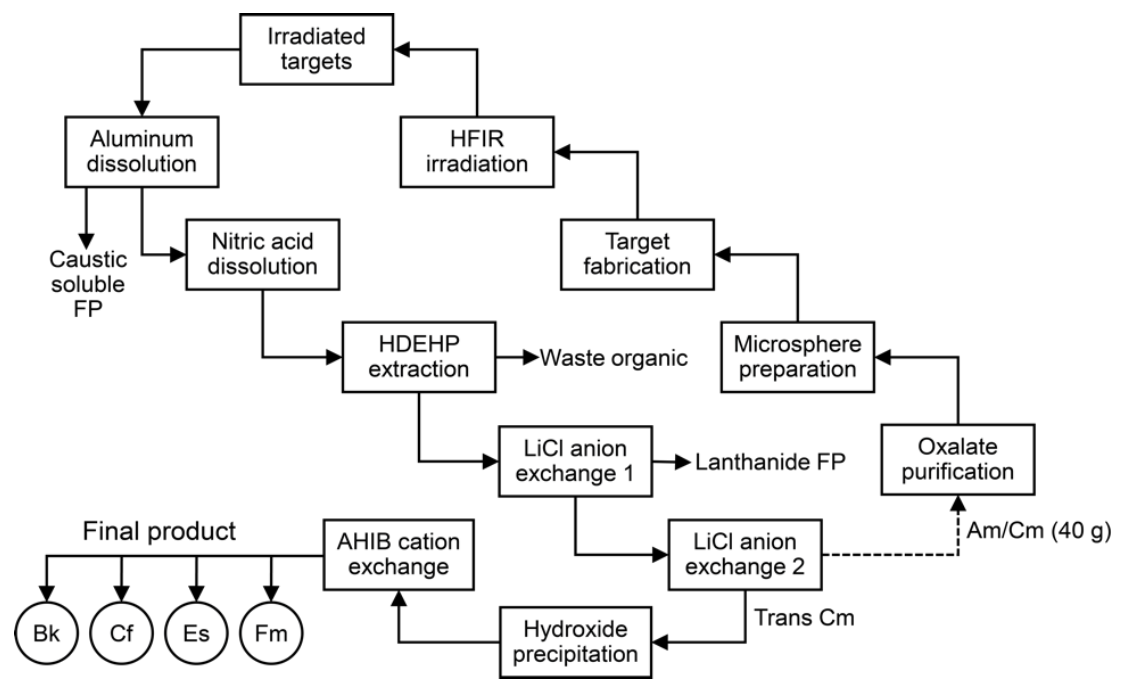

Figure 1. Process flow for production and separation of heavy actinides at HFIR/REDC. Starting with $40 \mathrm{~g}$ of $\mathrm{Am} / \mathrm{Cm}, \sim 200 \mathrm{mg}$ of ${ }^{252} \mathrm{Cf}$, 20mg of ${ }^{249} \mathrm{Bk}, 1-2$ micrograms of ${ }^{254} \mathrm{Es}$, and picograms of ${ }^{257} \mathrm{Fm}$ can be produced.

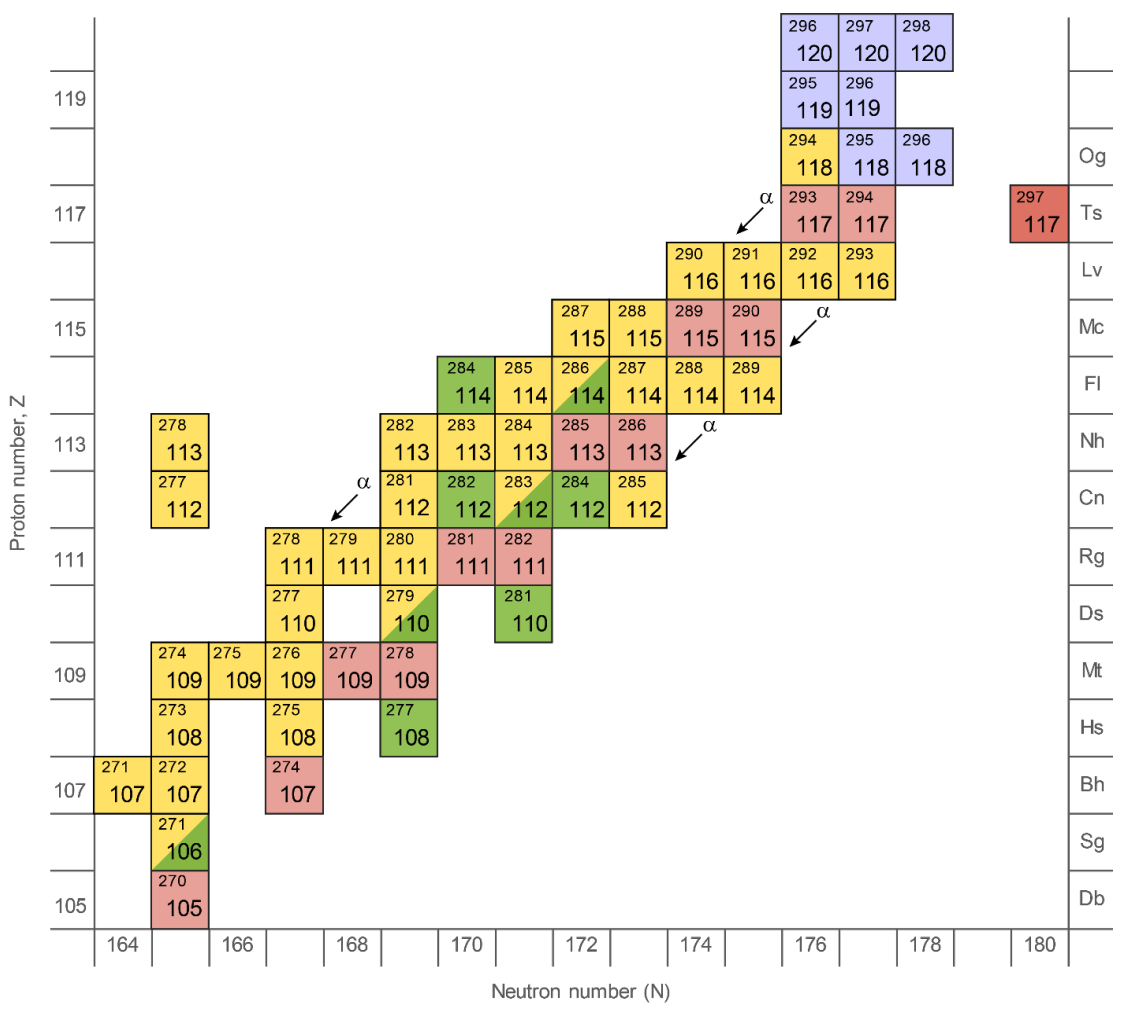

Figure 2. Nuclear landscape in the vicinity of isotopes of element 117 [18]. The compound nucleus ${ }^{297} 117$ (dark red) decays by emission of 3 or 4 neutrons to ${ }^{294} 117$ or ${ }^{293} 117$, respectively. Element 117 decay chains are shown in light red. ${ }^{278} 113$ and ${ }^{277} 112$ are the result of cold fusion reactions, all other isotopes were created in hot fusion reactions or decayed from hot fusion products. Blue denotes potentially accessible isotopes using current technology. (c) Taylor \& Francis Ltd., reproduced by permission of Taylor \& Francis, permission to reuse must be obtained from the publisher.

\subsection{Results}

A total of six atoms of element 117 were observed in 2010 [4], five of ${ }^{293} 117$ and one of ${ }^{294} 117$. These results were confirmed at GSI in Darmstadt, Germany [12] and in a second experiment at FLNR, JINR [13]. To date, 16 atoms of ${ }^{293}$ Ts and 6 atoms of ${ }^{294}$ Ts have been synthesized.
The alpha decay half-lives and energies for element 117 are summarized in Figure 3, together with earlier results for odd- $Z$ elements. The data form a self-consistent picture of nuclear behavior across five elements and 50 isotopes, with alpha half-lives increasing and alpha energies decreasing as neutron numbers increase, approaching the closed shell at $\mathrm{N}=184$. 
This provides strong evidence for the existence of the island of stability.

Also shown in Figure 3 are alpha half-life data for six isotopes of element $113(\mathrm{Nh})$ over a range of 8 neutron numbers, from ${ }^{278} \mathrm{Nh}$ (cold fusion results from multi-year experiments at RIKEN [6]) to ${ }^{282-286} \mathrm{Nh}$ (hot fusion results from JINR). Over this range the half-life increases by a factor of 6000 (from $1.4 \mathrm{~ms}$ to $9500 \mathrm{~ms}$ ) as the closed nuclear shell at $\mathrm{N}=184$ is approached. A similar range of neutron numbers for element 112 (copernicium) demonstrates an increase in the tens of thousands with increasing neutron number.
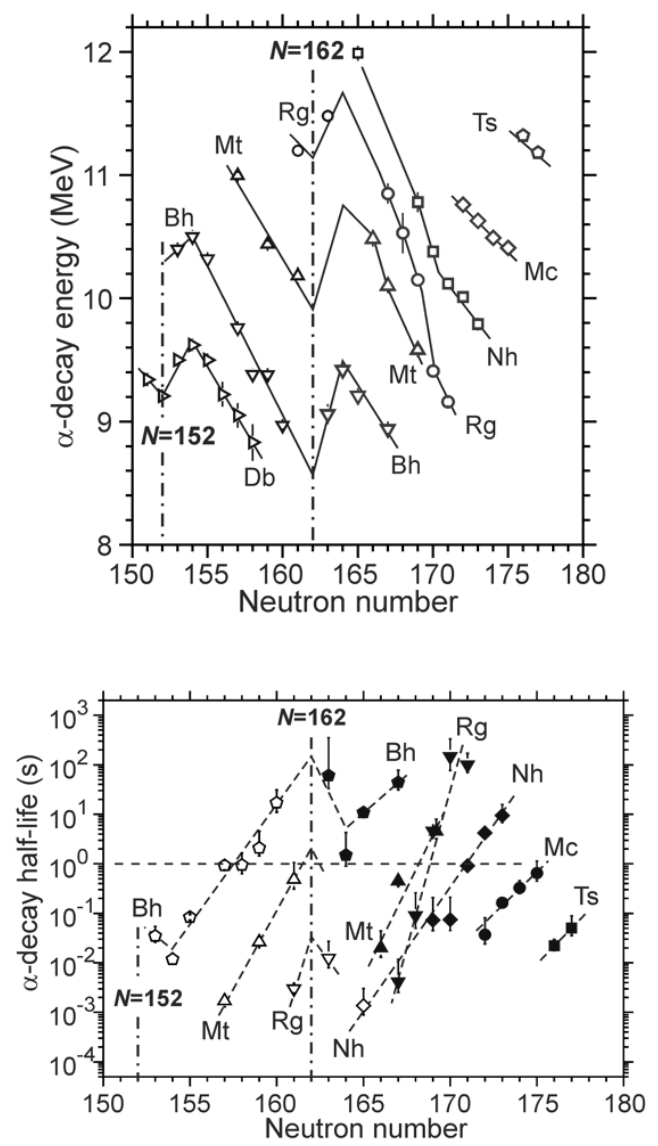

Figure 3. Alpha decay energies and half-lives for odd isotopes in the region of superheavy elements. Note the consistent trends in the data, including decreases in energies and increases in half-lives for $\mathrm{Nh}, \mathrm{Mc}$, and Ts isotopes as the closed shell at $\mathrm{N}=184$ is approached. Similar trends are observed for even isotopes [2]. (c) Yu. Ts. Oganessian and V. K. Utyonokov, reproduced by permission of the rightsholder.

\section{Summary and next steps}

A new chemical element, tennessine (Ts) has been discovered with atomic number $Z=117$, completing row 7 of the periodic table. Two isotopes of Ts were synthesized, ${ }^{293}$ Ts and ${ }^{294}$ Ts. ${ }^{294} \mathrm{Og}$ and ${ }^{294} \mathrm{Ts}$ are the heaviest nuclei observed to date. ${ }^{294} \mathrm{Ts}$, together with ${ }^{293} \mathrm{Lv}$, represent the closest approach to date to island of stability and the closed nuclear shell at $\mathrm{N}=184$. In addition to the two isotopes of Ts, ten new heaviest isotopes of elements 115,113,111, 109, 107, and 105 were observed in element 117 decay chains, all closer to the presumed island of stability. The results present a consistent picture of nuclear properties for superheavy elements and strong evidence for the existence of the island of stability.

Overall, the use of actinide $+{ }^{48} \mathrm{Ca}$ hot fusion reactions has led to the discovery of five new elements with $Z=114-118$. Going above $Z=118$ will require new beams (such as ${ }^{50} \mathrm{Ti}$ or ${ }^{51} \mathrm{~V}$ ) or new actinide targets heavier than $\mathrm{Cf}$ (such as Es). Production cross sections are expected to decline by a factor of ten or more with ${ }^{50} \mathrm{Ti}$ compared to ${ }^{48} \mathrm{Ca}$ and even more so for heavier beams such as ${ }^{51} \mathrm{~V}$ [14]. The situation is even less favorable for heavier targets, since current approaches yield only 1-2 micrograms of ${ }^{254} \mathrm{Es}$, when $10 \mathrm{mg}$ is typically required for an actinide target.

New approaches are needed to improve the probability of success of these experiments. New facilities being completed at the Superheavy Element Factory at JINR, as well as upgrades at RIKEN and GANIL and proposed new capabilities at GSI, offer the potential to increase production rates by one or two orders of magnitude, effectively compensating for the reduced production cross sections resulting from the use of heavier beams. Elements 119 and 120 appear to be within reach using ${ }^{50} \mathrm{Ti},{ }^{51} \mathrm{~V}$, or $54 \mathrm{Cr}$ with $\mathrm{Cm}, \mathrm{Bk}$, and Cf targets, as appropriate.

Another approach would be to develop new technology for increased production of ${ }^{254}$ Es. In the 1980s, a Large Einsteinium Activation Project (LEAP) was proposed [15] with the goal to increase the production of Es to 40 micrograms. This would be sufficient to prepare an Es target of approximately $0.1 \mathrm{~cm}^{2}$ in area at the appropriate thickness $(\sim 0.4$ $\mathrm{mg} / \mathrm{cm}^{2}$ ) for superheavy element production. The use of this target would require improved cooling technology as the target would be required to accept the entire beam continuously over this small area in order to maintain reasonable production rates. An ${ }^{254} \mathrm{Es}$ target, if available, would provide an attractive path to elements 119 and 121 using ${ }^{48} \mathrm{Ca}$ and ${ }^{50} \mathrm{Ti}$ beams, respectively, and could increase neutron number for element 119 by 3 compared to the proposed ${ }^{50} \mathrm{Ti}$ on ${ }^{249} \mathrm{Bk}$ approach.

Heavier isotopes of $\mathrm{Cf}$ (notably long-lived ${ }^{251} \mathrm{Cf}$ ) would increase neutron number for experiments involving $\mathrm{Cf}$ targets. ${ }^{251} \mathrm{Cf}$ can be obtained from decades-old $\mathrm{Cf}$ sources where the highly-radioactive ${ }^{252} \mathrm{Cf}$ has decayed away (2.6 year half-life). This leaves a remaining mixture of approximately $50 \%{ }^{249} \mathrm{Cf}, 15 \%$ ${ }^{250} \mathrm{Cf}$, and $35 \%{ }^{251} \mathrm{Cf}$. Mixed $\mathrm{Cf}$ material has been recovered from old $\mathrm{Cf}$ sources at REDC [16] and is being used in experiments at Dubna to search for the $\mathrm{A}=295$ and 296 isotopes of $\mathrm{Og}$ (element 118), which would be 1 or 2 neutrons heavier, respectively, than the current heaviest known nuclei, ${ }^{294} \mathrm{Ts}$ and ${ }^{294} \mathrm{Og}$.

Neutron number can also be increased by accessing the $2 \mathrm{n}$ channel in hot fusion reactions. Production cross 
sections are expected to decrease by about a factor of ten [17], in comparison to the $3 \mathrm{n}$ and $4 \mathrm{n}$ channels that have been typically used in superheavy element synthesis. The expected increases in beam currents at new and upgraded accelerator facilities make this a reasonable option for adding an additional neutron.

Radioactive ion beams, such as from the Facility for Rare Ion Beams (FRIB) currently under construction at Michigan State University, offer potential for significant increases in neutron number in the future. This will require significant increases in beam currents beyond currently available technology.

\section{Acknowledgements}

This research was supported by the U.S. Department of Energy (DOE) Office of Nuclear Physics, Isotope Development and Production for Research and Applications Program and Low Energy Nuclear Physics Program. The author is indebted to staff at the ORNL Radiochemical Engineering Development Center and High Flux Isotope Reactor, a DOE Office of Science User Facility, for their support in the production and chemical separation of the actinide materials.

The author gratefully acknowledges many colleagues at ORNL, the Flerov Laboratory of Nuclear Reactions (JINR, Dubna, Russia), Lawrence Livermore National Laboratory, GSI (Darmstadt, Germany), University of Mainz (Mainz, Germany), Vanderbilt University, and the University of Tennessee-Knoxville, without whom this research would not have been possible.

This manuscript is based on an invited presentation by the author at Actinides 2017, Sendai, Japan, July 10-14, 2017.

This manuscript has been authored by UT-Battelle, LLC under Contract No. DE-AC05-00OR22725 with the U.S. Department of Energy. The United States Government retains and the publisher, by accepting the article for publication, acknowledges that the United States Government retains a non-exclusive, paid-up, irrevocable, world-wide license to publish or reproduce the published form of this manuscript, or allow others to do so, for United States Government purposes. The Department of Energy will provide public access to these results of federally sponsored research in accordance with the DOE Public Access Plan: http://energy.gov/downloads/doe-public-access-plan

\section{References}

[1] L. Ohrstrom and J. Reedijk, Names and symbols of the elements with atomic numbers $113,115,117$, and 118 , Pure Appl. Chem. 88 (2016), pp. 12-17.

[2] Yu.Ts. Oganessian and V.K. Utyonkov, Superheavy nuclei from ${ }^{48} \mathrm{Ca}$-induced reactions, Nucl. Phys. A 944 (2015), pp. 62-98.

[3] J.B. Roberto et al., Actinide targets for the synthesis of superheavy elements, Nucl. Phys. A 944 (2015), pp. 99-116.

[4] Yu.Ts. Oganessian et al., Synthesis of a new element with atomic number Z=117, Phys. Rev. Lett. 104 (2010), 142502.

[5] Yu.Ts. Oganessian, Heaviest nuclei from ${ }^{48} \mathrm{Ca}$ induced reactions, Journal of Physics G 34 (2007), R165.

[6] K. Morita et al., Experiment on the synthesis of element 113 in the reaction ${ }^{209} \mathrm{Bi}\left({ }^{70} \mathrm{Zn}, \mathrm{n}\right)^{278} 113$, Journal of the Physical Society of Japan 81 (2004), pp. 2593-2596.

[7] I.W. Osborne-Lee and C.W. Alexander, Californium: A remarkably versatile radioisotope, ORNL/TM-12706 (1995).

[8] J.E. Bigelow, E.D. Collins and L.J. King, Actinide separations, J.D. Navratil and W.W. Schulz, eds., ACS Symp. Ser. 117, American Chemical Society, Washington, DC. (1980), pp. 147-155,

[9] E.D. Collins, D.E. Benker, F.R. Chattin, P.B. Orr and R.G. Ross, Transplutonium elements-production and Recovery, J.D. Navratil and W.W. Schulz, eds., $A C S$ Symp. Ser. 161, American Chemical Society, Washington, DC. (1981), pp. 147-160,

[10] D.E. Benker, F.R. Chattin, E.D. Collins, J.B. Knauer, P.B. Orr, R.G. Ross and J.T. Wiggins, Transplutonium elements-production and recovery, J.D. Navratil and W.W. Schulz, eds., ACS Symp. Ser. 161, American Chemical Society, Washington, DC. (1981), pp. 161-172,

[11] D. Hobart and J. Peterson, The Chemistry of the Actinide and Transactinide Elements, $4^{\text {th }}$ ed., L.R. Morss, N.M. Edelstein, J. Fuger, eds., Vol. 2, Springer (Netherlands). (2010), pp. 1444-1498,

[12] J. Khuyagbaatar et al., ${ }^{48} \mathrm{Ca}+{ }^{249} \mathrm{Bk}$ fusion reaction leading to element $\mathrm{Z}=117$ : long-lived alpha-decaying ${ }^{270} \mathrm{Db}$ and discovery of ${ }^{266} \mathrm{Lr}$," Phys. Rev. Lett. 112 (2014), 172501.

[13] Yu. Ts. Oganessian et al., Production and decay of the heaviest nuclei ${ }^{293,294} 117$ and ${ }^{294} 118$, Phys. Rev. Lett. 109 (2012) 162501.

[14] G.G. Adamian, N.V. Antonenko, A.N. Bezbakh and R.V. Jolos, Effect of properties of superheavy nuclei on their production and decay, Physics of Particles and Nuclei 47 (2016), pp. 387-455.

[15] J.E. Bigelow, C.W. Alexander and L.J. King, Proposed production of a large (40 microgram) sample of ${ }^{254} \mathrm{Es}$, Symposium on Heavy Element Research, AICHE Summer National Meeting, Seattle, Washington (1985).

[16] R.A. Boll et al., Electrodeposition of decay- enriched ${ }^{251} \mathrm{Cf}, 16^{\text {th }}$ International Workshop on Targetry and Target Chemistry, Santa Fe, New Mexico (August 29-September 1, 2016).

[17] K. Siwek-Wilczynska, T. Cap, M. Kowal, A. Sobiczewski and J. Wilczynski, Phys. Rev. C 86 (2012), 014611.

[18] J.B. Roberto and K.P. Rykaczewski, Discovery of element 117: superheavy elements and the island of stability, Separation Science and Technology, DOI:10.1080/01496395.2017.1290658 (2017). 